\author{
BRAZILIAN JOURNAL \\ $\mathrm{OF}$ \\ RADIATION SCIENCES \\ XX (2018) 01-10
}

\title{
Evaluation of gastric emptying by means of scintigra- phy using different test meals
}

\author{
Montoya $^{a}$ V., Quintão ${ }^{b}$ M. F., Marino ${ }^{b}$ V. S. P., Coelho ${ }^{c}$ L. G.V., Marinho ${ }^{c}$ F. P., \\ Mota $^{\mathrm{b}}$ L.G., Mourão ${ }^{\mathrm{a}, \mathrm{d}}$ A.P \\ ${ }^{a}$ Department of Nuclear Engineering, Federal University of Minas Gerais (UFMG) \\ Av. Pres. Antônio Carlos, 6627 CEP: 31270-901 Belo Horizonte, MG, Brazil \\ ${ }^{b}$ Department of Anatomy and Image, Federal University of Minas Gerais (UFMG) \\ Av. Prof. Alfredo Balena, 190 CEP 30130-100 Belo Horizonte, MG, Brazil \\ ${ }^{c}$ Gastroenterology ALFA Institute, Clinical Hospital, Federal University of Minas Gerais (UFMG) \\ Av. Prof. Alfredo Balena, 110, CEP: 30130-100, Belo Horizonte, MG, Brazil \\ ${ }^{d}$ Biomedical Engineering Center, Federal Center for Technological Education of Minas Gerais (CEFET-MG) \\ Av. Amazonas 5253, CEP 30421-169 Belo Horizonte, $M G$ \\ vimoquifa@yahoo.com.ar
}

\begin{abstract}
Gastroparesis is a disorder characterized by symptoms and evidence of delayed gastric emptying (GE) in the absence of mechanical obstruction. Scintigraphy has been considered a "gold standard" for measuring Gastric Emptying Time (GET) and retention percentage of radio-labeled food, after four hours of ingestion. To obtain the images, it is recommended, worldwide, the use of the Tougas diet $(255 \mathrm{kcal})$ as a standard meal. The objective of this study was to compare the GET and the percentage of retention in the time of $4 \mathrm{~h}$, obtained by scintigraphy using the Tougas diet $(255 \mathrm{kcal})$ and the proposed diet $(319 \mathrm{kcal})$. Thirty patients, both genders, aged 23 to 76 years, were selected, they presenting clinical symptoms suggestive of gastroparesis. These parameters were measured for the same patient, but in different days. The results showed that the diet fat content used to obtain GE of solid foods by scintigraphy did not influence the retention percentage results after 4 hours of ingestion of the radiolabeled food. However, the time of two hours is an important parameter to be observed in the result of this examination. In addition, the data suggest that the proposed diet may be more efficient for the classification of the severity of GE delay in patients with clinical signs suggestive of gastroparesis.
\end{abstract}

Keywords: Gastric emptying, Scintigraphy, Test Meals

ISSN: 2319-0612

Accepted: 2018-11-02 


\section{INTRODUCTION}

Gastroparesis comprises a set of clinical conditions characterized by a delay in the passage of food through the stomach and into the small intestine, without evidence of mechanical obstruction [1].

Since 1966, the scintigraphic method has been used for qualitative and quantitative evaluation of the first choice of gastric emptying (GE). It is a non-invasive and functional method and, therefore, capable of reproducing the physiology of GE.

In 2008, the scintigraphic method was standardized for GE evaluation of solids in consensus published by the American Society of Neurogastroenterology and Motility and the Society of Nuclear Medicine. In this consensus, the low-fat diet described by Tougas et al. (2000) to perform this examination [2], with images acquisition, immediately after its ingestion and at 60, 120, 180, and 240 minutes, translating GE by the percentage of radioactive activity (radioactive counts) retained in the stomach after 4 hours of the standard meal intake [3]. However, clinical evidence of postprandial entrapment in patients with normal GE scintigraphy performed with the Tougas diet [2], raised many doubt especially, because this diet did not reflect the usual feeding conditions of these patients, as pointed out by other authors $[4,5]$.

The objective of this study was to compare the GET and the percentage of retention in the time of 4 hours, obtained by scintigraphy using the Tougas diet $(255 \mathrm{kcal})$ and the proposed diet (319 kcal).

In order to evaluate the role of the diet, in this study, patients with a dyspepsia clinic stratified according to the adopted diet of Tougas [2] and with the inclusion of a higher fat content (diet proposed) were recruited, correlating them with the patients. The relevance of this study lies in the diet proposal that best reproduces the clinical condition of the patient, increasing the diagnostic and therapeutic accuracy.

\section{MATERIALS AND METHODS}


Thirty patients with clinical suspicion of gastroparesis treated at the Intestine Outpatient Clinic of the Alpha Institute of Gastroenterology of the Hospital das Clínicas of UFMG, Belo Horizonte, MG, Brazil, were consecutively selected. The inclusion criteria were: clinical symptoms suggestive of GE delay and those of exclusion: diabetes mellitus, peptic ulcer, gastric surgery and pregnancy. All patients performed solid GE scintigraphy, after 8 hours of fasting, using the Tougas diet and, in a second moment, the diet proposed in this study. None of the patients was on medication that interfered with GE. The solid diet of Tougas [6] consisted of an omelet made with two eggs, without the egg yolks, marked with ${ }^{99 \mathrm{~m}} \mathrm{Tc}$-colloidal tin $(74 \mathrm{MBq} / 2 \mathrm{mCi})$, two slices of bread, $30 \mathrm{~g}$ of strawberry jam, accompanied by $120 \mathrm{~mL}$ of water, resulting in a caloric content of $255 \mathrm{kcal}$. The proposed solid diet consisted of an omelet made with two whole eggs, marked with ${ }^{99 \mathrm{~m}}$ Tc-colloidal tin (74 MBq / $2 \mathrm{mCi}$ ), two slices of bread, $30 \mathrm{~g}$ of butter, accompanied by $120 \mathrm{~mL}$ of water, with a caloric content of $319 \mathrm{kcal}$.

The images were acquired using the Hawkeye ${ }^{\circledR}$ gamma camera equipped with Xeleris ${ }^{\circledR}$ processing station (GE Healthcare, Milwaukee Wisconsin, USA), in the anterior and posterior projections, immediately after and after 1, 2 and 4 hours of the intake of the radiolabeled diet, being $60 \mathrm{~s}$ by frame, $140 \mathrm{keV}$ window $\pm 20 \%$. The following parameters were estimated: Half-times $\left(\mathrm{T}_{1 / 2}\right)$ of emptying (reference value $\leq 100 \mathrm{~min}$ ) [7]; Radioactive activity in the stomach (content retained) as a percentage at 2 and 4 hours of ingestion (abnormal: $>60 \%$ at $2 \mathrm{~h}$ and $>10 \%$ at $4 \mathrm{~h}$ ) [3]; Delayed severity rating at $4 \mathrm{~h}$ (mild retention: $11-15 \%$, moderate retention: $16-35 \%$ and severe $>35 \%$ ) [4].

The results of $T_{1 / 2}$ of GE and retention of the radiolabeled food in the stomach were presented as mean and standard deviation. For rejection of the null hypothesis, the Shapiro-Wilk normality test was used. Wilcoxon's test for related non-parametric samples was used to compare the groups, and Student's t-Test was used for related parametric samples. The values of $p<0.05$ were considered statistically significant. All data were analyzed using SPSS® software version 24.

\section{RESULTS AND DISCUSSION}

Considering 30 patients who participated in the study, $26.7 \%$ were male and $73.3 \%$ female. The group age ranges from 23 to 76 years old, with a weighted average of $56.7 \pm 13.4$ years. 
It was found a difference in the percentage of gastric retention at $2 \mathrm{~h}$ between the two diets tested, with a higher precocity when using the proposed diet $(\mathrm{p}=0.001)$, not evidenced at $4 \mathrm{~h}(\mathrm{p}=0.1)$ - (Figure 1). According to Maurer (2015), the stomach and stomach regions present individual functions in the GE, some patients may present altered GE at $2 \mathrm{~h}$ and normal at $4 \mathrm{~h}$ and vice versa [3]. The early phase of GE analysis of solid foods by scintigraphy $(0-2 \mathrm{~h})$ reflects the action of the gastric fundus, while the late phase (2-4 h) reflects grinding by the antrum and propulsion of the food to the duodenum. According to Maurer (2015) and Cremonini (2004), if there is a delay of GE within 2 hours, the study may be interrupted, as this result truly confirms a delay in GE [3,8].

Our data showed that all patients with delayed GE at $2 \mathrm{~h}$ had a delay at $4 \mathrm{~h}$, without the need to maintain the evaluation until $4 \mathrm{~h}$. However, Bonta et al. (2011) reported that reducing the examination time to two hours represents a loss of sensitivity and reproducibility of the method [9]. Thus, the results found suggest that the proposed diet presents greater sensitivity for the diagnosis of retention or and to better reflect the alterations of the stomach as compared to the Tougas diet, allowing the evaluation of the specific therapeutic efficacy for fundus and / or antrum dysfunctions [3].

Figure 1: Percentage of retention of the radiolabeled diet in the stomach, with the two diets tested, in the times of 2 and 4 hours. $(* P<0.05)$.

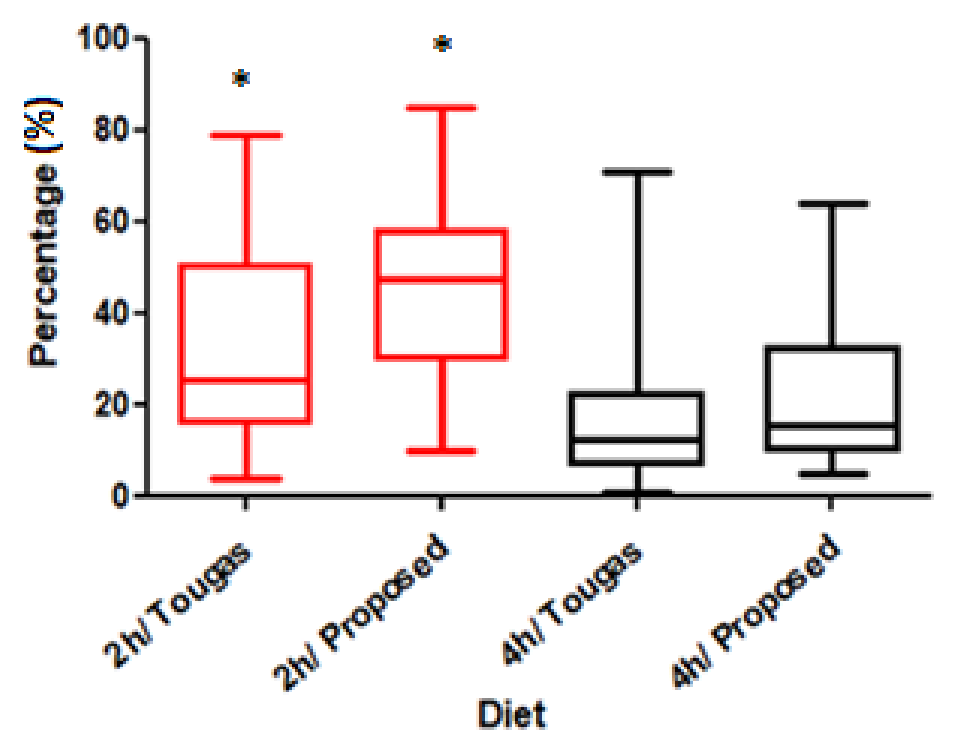


Table 1 illustrates the classification of the severity of GE delay according to the sex and type of diet administered, showing that in the Tougas diet the patients had a normal delay $(<10 \%)$ of $33.4 \%$ and a proposed diet had a delay of $30 \%$. It should be noted that study patients the severe delay was $10 \%$ as in the Tougas diet and a $23.4 \%$ in the proposed diet, it was able to point out a greater delay severity (almost three times more than the Tougas diet).

The majority of the patients who presented a delay in time of GE at $2 \mathrm{~h}$, presented a delay at $4 \mathrm{~h}$, classified as severe, suggesting that the marked delay may be due to changes in the stomach floor. This fact has clinical relevance since the treatment is based on the severity of the symptoms and the type of delay, that is, if early, it would be compatible with the antrum alterations and, late, when there are alterations in the background.

The choice of specific prokinetic drugs for each segment could optimize clinical treatment. In cases of exceptional severity, including those refractory to any therapeutic approach, the possibility of surgical treatment has been considered [10].

Table 1: Classification of the severity of the delay in the EG at 4 hours, according to diet and sex.

\begin{tabular}{|c|c|c|c|c|c|c|c|c|c|c|}
\hline & \multicolumn{5}{|c|}{ Tougas Diet (255Kcal) } & \multicolumn{5}{|c|}{ Proposed Diet (319Kcal) } \\
\hline & & Male & & male & Total & & Male & & male & Total \\
\hline & $\mathbf{N}$ & $\%$ & $\mathbf{N}$ & $\%$ & $\%$ & $\mathbf{N}$ & $\%$ & $\mathbf{N}$ & $\%$ & $\%$ \\
\hline Normal & 2 & 6.7 & 8 & 26.7 & 33.4 & 2 & $6.67 \%$ & 7 & $23.3 \%$ & $30 \%$ \\
\hline Light & 4 & 13.3 & 3 & 10 & 23.3 & 2 & $6.67 \%$ & 5 & $16.7 \%$ & $23.4 \%$ \\
\hline Moderate & 2 & 6.7 & 8 & 26.7 & 33.4 & 2 & $6.67 \%$ & 5 & $16.7 \%$ & $23.4 \%$ \\
\hline Severe & 0 & $0 \%$ & 3 & 10 & 10 & 2 & $6.67 \%$ & 5 & $16.7 \%$ & $23.4 \%$ \\
\hline Total & 8 & $26.67 \%$ & 22 & $73.4 \%$ & $100 \%$ & 8 & $26.67 \%$ & 22 & $73.4 \%$ & $100 \%$ \\
\hline
\end{tabular}

Table 2 shows the $\mathrm{T}_{1 / 2}$ values, according to the diet used. We analyzed 28 and 27 patients who received the Tougas diet and the proposal, respectively, excluding 5 patients for total $\mathrm{T}_{1 / 2}>240 \mathrm{~min}$, 
independently of the diet used and extrapolating the examination time. A higher value of $T_{1 / 2}$ was observed when the proposed diet was used. According to the Shapiro-Wilk test used for $\mathrm{T}_{1 / 2}$ analysis, the Tougas diet does not have a normal distribution of results $(\mathrm{p}<0.05)$, while the proposed diet has a normal distribution ( $p>0.05$ ). Therefore, by applying the Wilcoxon test, the $T_{1 / 2}$ values obtained by the two diets presented a statistically significant difference ( $\mathrm{p}<0.05$ ). Obviously, since the fat content between the two diets tested was different, it was expected that the proposed diet would have a slower $T_{1 / 2}$ because it contained a higher fat content, similarly affecting the percentage of retention of the radiolabeled food in time of $4 \mathrm{~h}$.

Table 2: $T^{1} \frac{1}{2}$ results obtained by scintigraphy with the two diets tested.

\begin{tabular}{cccccccc}
\hline & & $\mathbf{n} * \begin{array}{c}\text { obs* } \\
*\end{array}$ & Mean \pm SD & Min-Max*** & $\begin{array}{c}\text { Normality } \\
\text { Test (p) }\end{array}$ & p \\
\hline & $\begin{array}{c}\text { Tougas Diet } \\
(255 \mathrm{kcal})\end{array}$ & 30 & 28 & $85.91 \pm 44.36$ & $37.33-220.49$ & 0.0013 & \\
$\begin{array}{c}\text { Gastric } \\
\text { Emptying }\end{array}$ & & & & & & & \\
T 112 (min) & $\begin{array}{c}\text { Proposed Diet } \\
\text { (319kcal) }\end{array}$ & 30 & 27 & & $111.01 \pm 43.68$ & $43.58-218.77$ & 0.2789 \\
& & & & & \\
\hline
\end{tabular}

$* \mathrm{n}=$ Total number of patients in the study; $* *$ obs $=$ Patients considered in the statistical analysis of $\mathrm{T}^{1} \frac{1}{2} ; * * *$ Min-Max $=$ Minimum-Maximum.

According to the Consensus of the American Society of Neurogastroenterology and Motility and the Society of Nuclear Medicine, the result of GE scintigraphy of solid foods should be given by the retention value of the radiolabelled food, after $4 \mathrm{~h}$ of its intake [4]. This consensus does not recommend the quantification of EG using $\mathrm{T}^{1} / 2$ due to the irregular shape of the counting curves as a function of time, failure to provide information on the distribution of the food throughout the stomach over time and to be quantified by means of a single point on the curve in question [11].

Figure 2 illustrates the images from patient 3, where the results showed normal GE in the Tougas diet and GE delay in the proposed diet. Figure 2A shows the retention of the radiolabelled food in the stomach at the initial time (Time 0) with adequate elimination from the stomach after $4 \mathrm{~h}$ of 
ingestion (5\% retention). However, with the proposed diet, this patient presented delay of elimination of the meal used after $4 \mathrm{~h}$, with retention of $12 \%$ of the material in the stomach antrum (Figure 2B).

Figure 2: Scintigraphic images using the Tougas diet (A) and the diet proposed $(B)$ of the patient 3. The arrows indicate retention of the radiolabelled food in the stomach antrum, in the anterior and posterior projections, after 4 h of ingestion. 


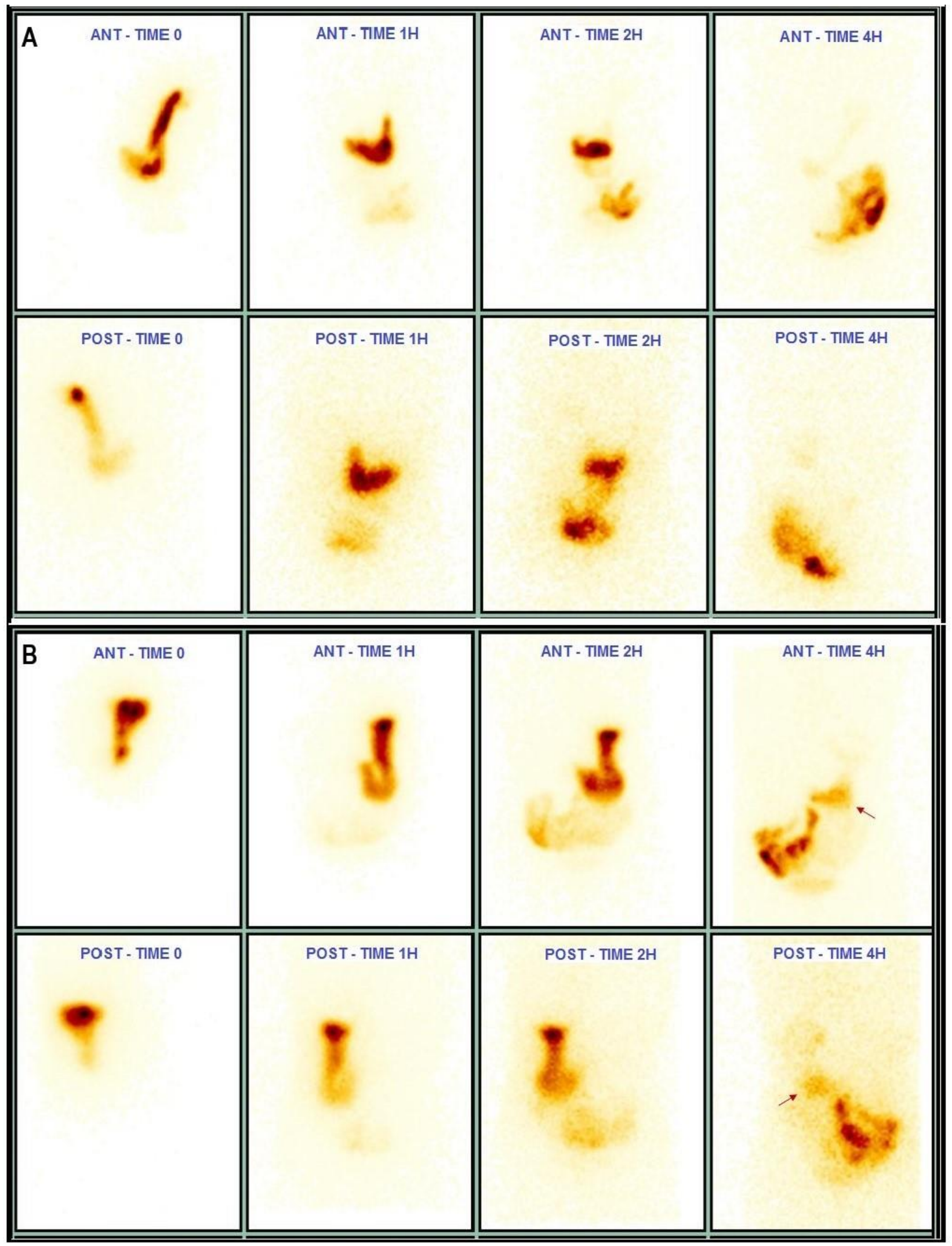


The scintigraphic images of patient 2 showed a delay of GE in the two diets tested. However, with the proposed diet, the delay was considered severe (39\%) (Figure 3). It can be observed in the diet of Tougas (Figure 3A), accumulation of the radiolabeled food in the antral region of the stomach after $4 \mathrm{~h}$ of ingestion (retention of 23\%). Using the proposed diet (Figure 3B), one can notice the accumulation of the meal used throughout the stomach region.

Figure 3: Scintigraphic images employing the Tougas diet (A) and the diet proposed (B) of patient 2. The arrow indicates accumulation of the radiolabelled food in the stomach antrum region after 4 h of ingestion. 


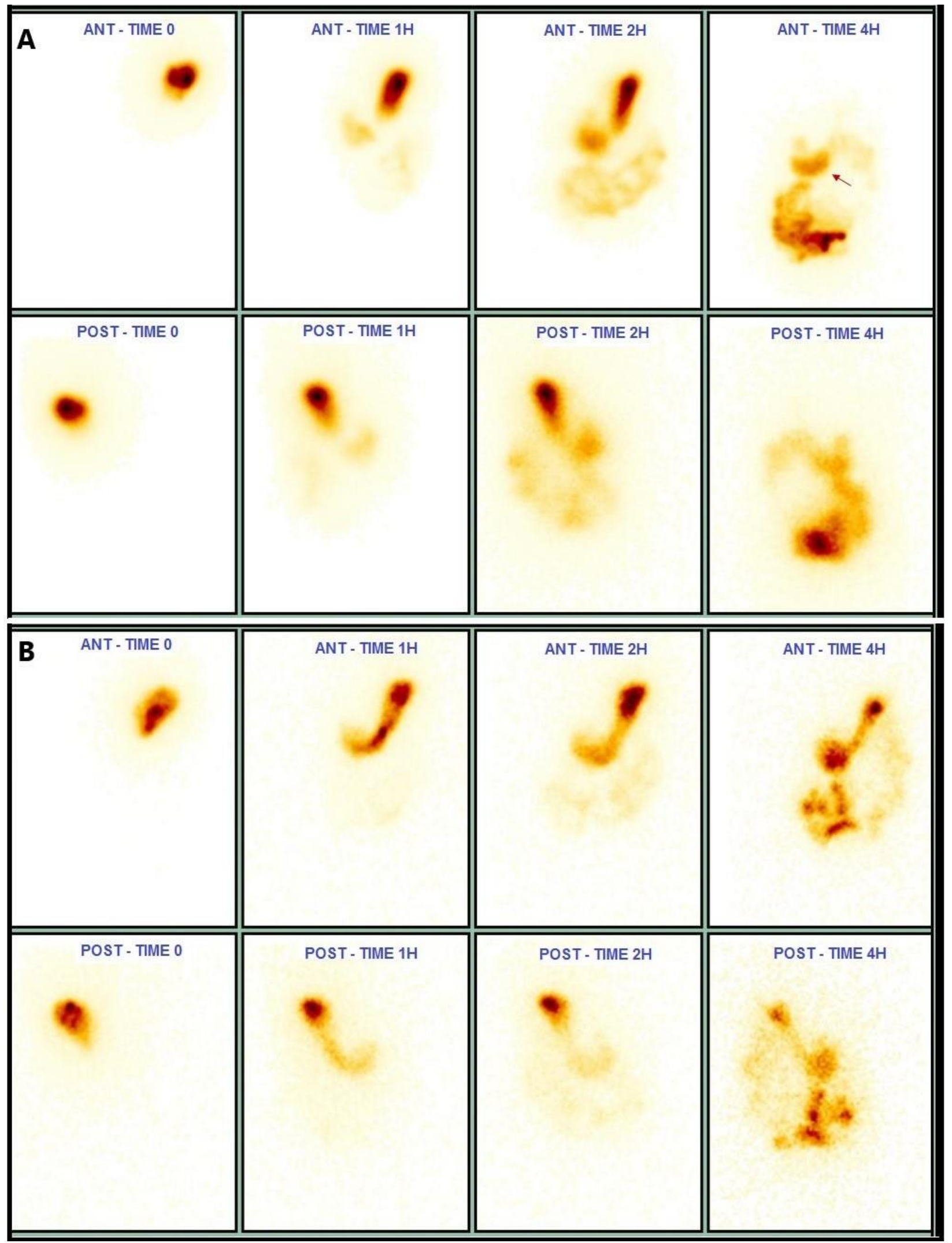




\section{CONCLUSION}

Obtained results hadn't shown a statistically significant difference between the two analyzed diets for percent retention of radiolabelled food after $4 \mathrm{~h}$ ingestion. Therefore, contrary to the consensus of the Nuclear Medicine Society and American Neurogastroenterology and Motility Society, the variation of the fat content in the test used did not influence the results of gastric emptying of solid food.

The two hour time after ingestion of the radiolabelled diet have shown a great importance in the evaluation of the EG of solid foods, reflecting the kinetics of GE and the stomach area affected.

The severity of the delay was better evidenced using the proposed diet, with a higher fat content, this fact may be useful as a tool for clinical treatment.

\section{REFERENCES}

[1] HASLER, W.L. Gastroparesis: pathogenesis, diagnosis and management. Nat Rev Gastroenterol Hepatol, v.8, p.438-453, 2011.

[2] TOUGAS, G.; EAKER, E.Y.; ABELL, T.L.; ABRAHAMSSON, H.; BOIVIN, M.; CHEN, J.; HOCKING, M.P.; QUIGLEY, E.M.M.; KOCH, K.L.; TOKAYER, A.Z.; STANGHELLINI, V.; CHEN, Y.; HUIZINGA, J.D.; RYDÉN, J.; BOURGEOIS, I.; MCCALLUM, R.W. Assessment of gastric emptying using a low fat meal: establishment of international control values. The American Journal of Gastroenterology, v. 95, p. 1456-1462, 2000a.

[3] MAURER, A.H. Gastrointestinal Motility, Part 1: Esophageal Transit and Gastric Emptying. Journal of Nuclear Medicine, v. 56, p. 1229-1238, 2015.

[4] ABELL, T.L.; CAMILlERI, M.; DONOHOE, K. A Consensus recommendations for gastric emptying scintigraphy: a joint report of the American Neurogastroenterology and Motility Society and the Society of Nuclear Medicine. American Journal of Gastroenterology, v. 102, p. 1$11,2008$. 
[5] LIN, H.C.; VAN CITTERS, G.W.; ZHAO, X.T.; WAXMAN A. Fat intolerance depends on rapid gastric emptying. Digestive Diseases and Sciences, v. 44, p. 330-335, 1999.

[6] TOUGAS, G.; EAKER, E.Y.; ABELL, T.L.; ABRAHAMSSON, H.; BOIVIN, M.; CHEN, J.; HOCKING, M.P.; QUIGLEY, E.M.M.; KOCH, K.L.; TOKAYER, A.Z.; STANGHELLINI, V.; CHEN, Y.; HUIZINGA, J.D.; RYDÉN, J.; BOURGEOIS, I.; MCCALLUM, R.W. Assessment of gastric emptying using a low fat meal: establishment of international control values. The American Journal of Gastroenterology, v. 95, p. 1456-1462, 2000 b.

[7] DICKMAN, R.; STEINMETZ, A.; BERNNSTINE, H.; GROSHAR, D.; NIV, Y. A novel continuous breath test versus scintigraphy for gastric emptying rate measurement. Journal of Clinical Gastroenterology, v. 45(1), p. 22-25, 2011.

[8] CREMONINI, F.; DELGADO-AROS, S.; TALLEY, N. Functional dyspepsia: drugs for new (and old) therapeutic targets. Best Practice \& Research Clinical Gastroenterology, v. 18, p. 717-733, 2004.

[9] BONTA, D.V.; LEE, H.; ZIESSMAN, H. Shortening the 4-hour gastric-emptying protocol. Clinical Nuclear Medicine, v. 36, p. 283-285, 2011.

[10] TRONCON, L.E.A. Gastroparesias: revisão de aspectos relacionados ao conceito, à etiopatogenia e ao manejo clínico. Revista da Associação Médica Brasileira, v. 43(3), p. 228-236, 1997.

[11] PODCZECK, F.; NEWTON, J.M.; YUEN, K.H. The description of the gastrointestinal transit of pellets assessed by gamma scintigraphy using statistical moments. Pharm. Res, v. 12(3), p. 376-379, 1995 